\title{
Equilibrium models in multimodal container transport systems
}

\author{
Francesco Corman ${ }^{1,2} \cdot$ Francesco Viti ${ }^{3}$. \\ Rudy R. Negenborn ${ }^{1}$
}

Published online: 4 September 2015

(C) The Author(s) 2015. This article is published with open access at Springerlink.com

\begin{abstract}
Optimizing the performance of multimodal freight transport networks involves adequately balancing the interplay between costs, volumes, times of departure and arrival, and times of travel. In order to study this interplay, we propose an assignment model that is able to efficiently determine flows and costs in a multimodal network. The model is based on a so-called user equilibrium principle, which is at the basis of Dynamic Traffic Assignment problems. This principle takes into account transport demands to be shipped using vehicles that transport single freight units (such as trucks) or multiple freight units (such as trains and barges, where demand should be bundled to reach efficient operations). Given a particular demand, the proposed model provides an assignment of the demand over the available modes of transport. The outcome of the model, i.e., the equilibrium point, minimizes users' generalized costs, expressed as a function of mode, travel time and related congestion, and waiting time for bundling sufficient demand in order to fill a vehicle. The model deals with these issues across a doubly-dynamic time scale and in an integrated manner. One dynamic involves a learning dynamic converging towards an equilibrium (day-to-day) situation, reflecting the reaction of the players towards the action of the others. Another dynamic considers the possible departure time that results in minimum expected costs, also due to the fact that players mutually influence each other on the choice of departure times, due to congestion effects and costs for early/late arrival of freight units. This is a choice within a given time horizon such as a day or a week. We present a study on the influence and
\end{abstract}

$\triangle$ Francesco Corman

f.corman@tudelft.nl

1 Department of Maritime and Transport Technology, Delft University of Technology, Delft, The Netherlands

2 Center for Industrial Management, Katholieke Universiteit Leuven, Louvain, Belgium

3 Faculty of Science Technology and Communication, University of Luxembourg, Walferdange, Luxembourg 
sensitivity of different model parameters, in order to analyse the implications on strategic decisions, fostering a target modal share for freight transportation. We also study under which conditions the different modes can be substitutes for each other.

Keywords Multimodal transportation planning - Equilibrium model - Dynamic Traffic Assignment · Bottleneck model · Day-to-day dynamics

\section{Introduction}

Freight transport is an important building block of a supply chain, and a key process for reducing costs and environmental emissions for logistic systems. This relates mostly to the choice of mode to be used (continuous transport system, truck, train, vessel, plane, ...) and its speed and reliability. Different modes have different issues related to the possibility to reach a final destination at a preferred time. Due to the raising importance of just-in-time production and delivery, a reliable and timely delivery is of crucial importance.

The freedom offered by transporting freight over road by truck is an extremely valued asset for logistics networks. However, excessive truck usage, on traffic networks that are already heavily used by passenger traffic, causes the emergence and propagation of congestion, which decreases the attractiveness of transport over roads. Other disadvantages relate to the environmental emissions, and economies of scale, especially if Less-than-Full-Load shipments are considered. For the latter, sharing and consolidation concepts have been often presented to companies as an effective way for solving energy and emission problems, and improve economic efficiency. From a general perspective such approaches also result in an effective way to reduce the number of vehicles on the road, contributing to relieving of networks from congestion, and in turn saving fuel and reducing pollution. For this reason, policies are steadily discouraging truck distribution in favour of railway and waterway distribution, which are seen as more environmentally sustainable (European Commission 2001).

Beyond political and social reasons, the modal split in freight transport (i.e., the division of freight over different modes of transport) is a result of economic factors. It is therefore necessary to internalize the factors related to policy measures within the decision-making process of transport companies in order to achieve desired sustainable behaviour. Hence, in this study we develop a model that is able to study multimodal networks and determine factors leading to the given modal share, which might, for instance, identify policies that favour rail and barges to trucks. To this end, we address the problem of assigning freight flows to multimodal freight transport networks. This involves adequately balancing the interplay between costs, volumes, times of departure and arrival, and times of travel between different modes that have rather different characteristics. In order to study this interplay, we propose a so-called equilibrium model that is able to efficiently determine flows and costs in a multimodal network. Equilibrium models are widely used in car traffic analysis to study the outcome of a variety of choices (departure times, modes, routes) and the resulting costs for a set of travellers. The basic assumption that such models rely on 
is the assumption that every traveller will try to minimize a generalized cost function, and choose the option that is best for him/her, given that the other travellers will keep their own decision (Wardrop 1952). Equilibrium models can model and forecast the choices of travellers and resulting performance of a transport system in scenarios in which multiple factors and planning and management measures interact, making the response of the system not easily predictable.

In this paper we adopt this classical transportation modelling paradigm in the context of mode choice in freight transport. The innovative feature of the approach is the consideration of multiple scales of time dynamics. One time scale involves a learning dynamic over a long number of rounds (days); the other time scale involves a departure time choice within a given time horizon (within-day). The combination of these scales results in what can be called a within-day dynamic intertwined with a day-to-day equilibrium in Dynamic Traffic Assignment terms (Tampère et al. 2010). We are therefore able to consider an equilibrium process that goes beyond traditional static equilibrium approaches and that moreover includes variations over time, i.e. different modes can have different attractiveness at different times compared to a preferred arrival time at destination. In the case of freight transport, this attractiveness may also depend on the loading rate of each mode in a certain time interval. This is a decisive step forward compared to static freight models (such as those mentioned in Friesz et al. 1986; Guelat et al. 1990; Cantarella 1997) and has paramount importance in demand-responsive logistic services, which strongly vary their costs in time, and are characterized by different time-dependent parameters and constraints. One typical constraint is congestion: the travel time of all travellers depends on the decision of all travellers. We also include the relevant issue that some modes characterized by vehicles with large capacity of freight units, such as barges or trains, are aggregating demand, therefore exploiting economy of scale principles. This translates further in the need to fill the vehicle, resulting in a further dynamic over time.

Thus, the contributions of this paper are the following:

1. We define an equilibrium model (in Wardrop terms) able to deal with multiple time scales, namely a day-to-day dynamic (i.e., the convergence towards an equilibrium assignment) and within-day dynamics, i.e. the choice of departure time so to minimize generalized costs;

2. We target multimodal transportation and consider discrete freight units such as containers as means to utilize and/or share transportation services from a point of origin to a specified destination;

3. For modes that carry multiple freight units, a collaborative decision process is considered, i.e. a sufficient demand has to be bundled before a vehicle is actually travelling. Vehicles are supposed to leave with Full Load. For modes carrying a single freight unit, a vehicle can depart at any time;

4. For all modes different expressions of congestion are taken into account, relating to simple cost-flow functions [e.g., the well-known BPR-function (Bureau of Public Roads 1964) for road links] or a queuing model with a fixed amount of slots (for railway and barge links); 
5. We evaluate the approach over a small theoretical network with multiple modes, analysing sensitivity of the results to various parameters and incentives that policy makers may consider.

The remainder of the paper is organized as follows. We review the literature in freight planning, assignment, equilibrium models and multi-scale dynamics approaches in Sect. 2. Section 3 presents a detailed and formal description of the investigated problem, and the assumptions made. Section 4 proposes a methodology and a mathematical model describing the equilibrium situation. Section 5 proposes an algorithm to find the solution of the equilibrium problem when multi-scale dynamics and multiple modes are considered. The proposed approach is evaluated using experiments for a theoretical network in Sect. 6, and discussed in its applicability in Sect. 7. Finally, Sect. 8 concludes the paper and provides directions for future research.

\section{Literature review}

Here we briefly review the main issues in freight transport. For a general overview of optimization approaches in freight transport routing and planning, we refer to the recent survey in SteadieSeifi et al. (2014). There, the transport problem is divided into multiple stages, of which the tactical planning relates to the choice of links, modes, and assignment of freight volumes over links in terms of itinerary and frequency. The vast majority of the solution approaches reported does not include stochastic or dynamic aspects at this stage. Those dynamic aspects, or real-time requirements, are normally taken into account only for operational control. This last problem is generally addressed by very complex online scheduling and vehicle routing problems (see e.g., Bock 2010).

\subsection{Freight assignment}

The problem of assigning freight flows to single-mode and multi-mode networks has been studied, see for instance the works of Friesz et al. (1986), Guelat et al. (1990) and Cantarella (1997). Those problems are all solved within a static setting, i.e., the adopted cost-flow functions are time-independent and no departure-arrival time choice is considered. This assumption limits the possibility to evaluate the performance of the system especially in problems including scheduled processes with arrival time windows.

In reality, the application of assignment solutions to freight networks is also common in the context of seaways for freight. The amount of traffic planned by liner shipping companies has been modelled with equilibrium models by Bell et al. (2011). A general overview of the applicability of optimization and assignment methods for container liner shipping is presented further in the recent review by Khoi Tran and Haasis (2013). More recently, Bell et al. (2013) looked at the minimization of expected costs in assignment models for maritime networks. Assuming that routes and service frequencies are given, operating costs are fixed. 
The optimization then results in assigning routes to containers so that handling costs, container rental and inventory costs are minimized. Infrastructure capacity in port as well as in routes are included using linear constraints.

The extension to consider multiple modes and multiple geographical scales is addressed among others by Newton (2008). This increase in complexity is justified by the fact that multimodal transport is typically associated with longer distances and international flows. A freight demand model WORLDNET is proposed that describes a long distance, multimodal origin-destination matrix, and a network model that covers Europe, its neighbours, as well as intercontinental routes, both maritime and air cargo. A distribution model is applied to subdivide the trade flows. A multimodal assignment procedure based on a mix of stochastic multi-class user equilibrium for road, stochastic assignment for railways and so-called all-or-nothing (which assigns flows to the cheapest combination of links) for maritime services is then used to assign the flows to the transport modes.

The applicability for freight flows of assignment models originally developed for passenger traffic is discussed by Jourquin and Limbourg (2006). They report on different assignment techniques, namely the all-or-nothing, and more sophisticated static assignment approaches, which consider also congestion or infrastructure capacity constraints. Those latter approaches refer to the limited capacity of links, typically captured by adding time penalties when the volumes of traffic on links surpass certain levels (corresponding to a saturation flow). At the operational level, an all-or-nothing approach is typically used to assign container flows in the intermodal freight transport planning. This approach assigns the entire volume of the transport demand to the route with the minimum value of the user-supplied objective function, and normally refers to unlimited capacity of links. Consideration of infrastructure capacity can be inserted by an incremental heuristic approach that increases the flow along the intermodal route with the minimum costs until infrastructure capacity will be reached. Next, the next best candidate intermodal route can be considered, until the transport demand is completely served, in a typical multi-commodity flow perspective. Such a solution can easily be represented as a greedy algorithm that assigns flows incrementally, but it can deal in a very limited manner with variable costs and traffic conditions, leading in general to higher costs when put into practice. This issue can be solved by multimodal traffic assignment methods, which consider different modes as virtual links generalizing a connection between two points. Jourquin and Limbourg (2006) finally conclude that the traditional four-steps model (i.e., forecasting based on trip generation, trip distribution, mode choice and route assignment) used in traditional planning studies, the all-or-nothing assignment, and the simple equilibrium assignment are still unable to give adequate solutions; more sophisticated approaches must be explored. In this paper, we explicitly address this issue, by providing approaches in which costs and attractiveness of links are explicitly dynamic, and vary over time as a consequence of choices of other users, and own choices. We moreover go beyond the all-or-nothing assignment by considering a multi-path assignment, and a description of link capacities completely in line with their study.

A similar discussion of innovative traffic assignment is proposed in the recent work of Maia and do Couto (2013). The authors take into account infrastructure 
capacity constraint, and variable perception of costs between users. In typical traffic assignment only complex stochastic assignment models could deal with both requirements at the same time. To solve this issue, the authors use a step-wise loading algorithm, that considers freight in multiple categories. One at a time, a category is routed and loaded on the network in an incremental way, considering the congestion effects resulting from the categories already routed and loaded. This corresponds quite naturally to a situation in which some players have a stronger role than others (for instance, leaders versus followers, long-distance transport versus local, or old versus new to the market). The authors of this last work join many others in stating that passenger car traffic assignment has been receiving much attention lately and that its applicability to freight transport by assigning flows to virtual networks and virtual links has started only more recently, see the works by Harker (1987), Jourquin and Beuthe (1996) and Tavasszy (1996).

In passenger transport, and more specifically in vehicular traffic systems, traffic assignment is usually formulated as a set of criteria through which the demand for mobility is distributed over the links of a transport network. In this application domain, the impact of time-dependent costs and flows is an essential element to study, for example to include re-routing and/or re-scheduling strategies to avoid congestion. The class of Dynamic Traffic Assignment (DTA) models deals explicitly with such time-dependent dynamics, and overall to describe temporal distributions of demand and supply. Traffic dynamics are in DTA explicitly regarded by modelling opportunely the propagation of flows along the links in the network and their interaction at nodes. Decision making dynamics about departure time, modes are considered through various response functions based on behavioural and economic principles. Tampère et al. (2010) report that the complexity of DTA predominantly lies in finding a convenient trade-off between mathematical rigorousness and realistic traffic and behavioural models.

The advantage of adopting DTA in this study is its property of dealing with the time- and flow-dependency of the costs, i.e., delays due to queuing phenomena are considered explicitly in the propagation of the flow, hence allowing to calculate expected arrival times at the destination, and in turn to identify optimal departure times, consistent with the scheduled arrival time windows. In the following we focus on the DTA literature concerning collaborative modes, as the whole literature on this domain is vast. For an overview one can refer to, e.g., Peeta and Ziliaskopoulos (2001) or Viti and Tampère (2010).

\subsection{Dynamic assignment for collaborative modes}

Vehicular traffic assignment normally assumes individual non-cooperative drivers and decision makers. Collaborative modes instead refer to the need of coordinating multiple players before a trip is actually done. The advantage of collaborative modes (i.e., sharing a vehicle with multiple travellers) is the possibility to increase occupancy rates of vehicles and to reduce the total number of vehicles for the same distances travelled. The resulting system is very attractive in terms of reduced congestion, increased vehicle occupancy and therefore more environmentally friendly. 
The model developed and analysed in this paper has strong analogies with the field of dynamic ride-sharing, in which an agreement and a synchronization between multiple travellers is required in order to start a trip. Dynamic Ride Sharing (DRS) is the process by which some passengers do not take a car to reach their destination but instead rely on taking a lift from some casual travellers that happen to take a car and have available space for the same link. Despite the obvious potential economic gains offered by this solution, and the adoption of many policies and incentives in the past to foster its use, very few systems have been put into practice; such systems are still far from reaching the modal share sought. This issue is reviewed in detail in recent works such as Agatz et al. (2012) and Furuhata et al. (2013).

Equilibrium of ridesharing networks have been studied in the context of deterministic and stochastic user equilibria (Huang et al. 2000), or using a traditional bottleneck model (Qian and Zhang 2011). A factor still overlooked is the departure time choice as function of the so-called matching rate, which has a direct impact on the travel time experienced by people and thus their decisions. This has a direct link in the freight transport world with the need to bundle demand before a vehicle with large capacity can depart.

An active and complex field of research is moreover targeting intelligent algorithms that can match travellers that will share a ride, based on their desires and characteristics. Those matching problems are similar in practice to operational planning problems for freight as categorized by SteadieSeifi et al. (2014). The development of optimization algorithms capable of handling the complexity of matching rides is studied in Ghoseiri et al. (2011). On the other hand, the impact on travellers' behaviour and on transport system costs is relatively unexplored; this is, however, a critical issue when introducing such models for freight transport assignment.

In recent research, we have developed a basic link assignment model to describe ridesharing for traffic (Viti et al. 2012). We have also investigated equilibria, considering the time-dependent nature of scheduling the ride matches. We have shown in Viti and Corman (2013) that a region in the space of (travel time costs, distance-related costs, and fixed costs) exists where shared-mode services can compete with private vehicles, if the parameters determining mode choices are opportunely chosen. In addition to this, the exploratory work of Viti and Corman (2014) shows that by extending this single-dynamic model to account for within-day dynamics, an emerging behaviour under congested conditions can be found: users tend to increase their preference for participating in ridesharing services at the expense of shifting their departure times to earlier time periods with respect to drivers using a vehicle without sharing it.

\subsection{Discussion}

In this paper we make a step forward by bridging the gap between such an advanced traffic assignment and the world of freight transport assignment. In fact, the need for more sophisticated assignment methods has been put forward as an issue by many researchers in the academic community (see Jourquin and Limbourg 2006). The model proposed in this paper can be seen as an extension of Vickrey's departure 
time choice model (Vickrey 1969), which includes the scheduling of modes with bundling effects, and congestion in different modes.

We base our analysis on a user equilibrium principle, and take into account vehicles that both transport single freight units (such as trucks) as well as multiple freight units (such as trains and barges, where demand should be bundled together to reach efficient operations). We refer to a time-responsive freight network, where the time of arrival influences operational costs. The time of arrival is further a function of the time of departure (a direct decision by the players), and the travel time (an emerging value consequence of the choice of all other players, for what concerns congestion, availability of vehicles, service frequency, etc.). This structure is based on a distinct and precise representation of multiple time-scales, in contrast with the simplicity of freight assignment models in the literature.

In the remainder of this paper, we refer to modes that require bundling of demand as collaborative modes, in the sense that multiple transport demand units (equivalent to users in our case) need to collaborate and find a match to have the transport vehicle full and operated. This can reflect a variety of situations that include companies that have their own distribution vehicles, and would like to achieve economies of scale by offering the empty spots; as well as companies that do only forwarding and distribution tasks, such as third party logistics companies. Thus the approach is generic in the sense that multiple dynamic characteristics of supply chain systems, such as collaboration and pooling, can be modelled via these mechanisms.

We assume that cooperation between players may result in better choices for the users and the system, but we consider only the former as being the driving force of the change. In fact, we do not consider the fully-cooperative setting that is often assumed by mathematical optimization approaches aiming at improving at once all links of the supply chain. Instead, all players compete on the available transport supply to reach the minimum transport cost. All those aspects make it an innovative approach with regards to the freight assignment perspective, as well as in the general traffic assignment world. This work is an exploratory study that aims at determining the possibility and applicability of such an approach to freight transport networks.

\section{Problem statement}

Given a multimodal transport network with a set of modes $M$, and given a demand of discrete units of freights to be transported from an origin to a destination, the problem we study consists of assigning the demand to the modes in order to minimize total generalised costs.

We identify the freight units with the users to ease the comparison with user equilibrium principles. We also consider all users as independent, and all of them having a single transport unit. Those conditions may be relaxed in future research. We assume in this study that the demand for trips is constant and that all the users will make their trip, no matter how high the cost is. This assumption can be changed by altering the model, for example by changing the demand from constant to elastic. 
Costs are in general a function of the time at which the destination node is reached (i.e., delays are penalized, and early arrivals with respect to preferred arrival time are also slightly penalized); the distance related costs that depend on the travel time, mode, and amount of transport units carried, plus extra factors that may incentivize a particular mode.

We use Fig. 1 to introduce the problem. Three modes are possible: barges, with a specified large vehicle capacity in terms of transport units; rail, with a large vehicle capacity, but smaller than barges; and trucks. The latter mode has a vehicle capacity of a single transport unit. The three modes use different independent links.

A role has to be defined for modes using vehicles with a higher vehicle capacity than a single discrete unit. In fact, a train or a barge can be hired to transport a large amount of demand available, or an existing demand can be added in order to fill up an existing train and save costs. This requires the definition of a role, within the freight transport system. We refer to an active role if the demand is actively associated with the generation and management of the vehicle: the traveller takes initiative and would be able to travel by himself/herself if required. This for instance relates to organizing the departure of a vehicle, such as a train or a barge, and being the first one to load it. The vehicle, though, might still need more transport units to be actually profitable. A passive role is instead the one of somebody who "jumps on" an existing vehicle, this latter organized and started by some "active" role. From a real-life point of view, one can think of active roles as forwarders or shipper companies or third party logistics companies, while passive roles are customers of such companies. By distinguishing between active roles (associated to the amount of vehicles in the system) and passive roles (corresponding to transport demand, but not to vehicles), the model is able to seamlessly consider the amount of vehicles as a decision variable. This is the inherent result of the decision of the players, rather than a fixed a priori decision.

An assignment is the tuple of (mode; a role; and a departure time) for all users/ freight transport units in the system. The assignment is composed of and completely determined by the choices of users. Typical, each user tries to find the decision (i.e., his/her own assignment) that minimizes his/her own cost, given the decision of the others (i.e., their assignment). The equilibrium assignment is the special assignment that corresponds to the situation in which no user has incentive to change any

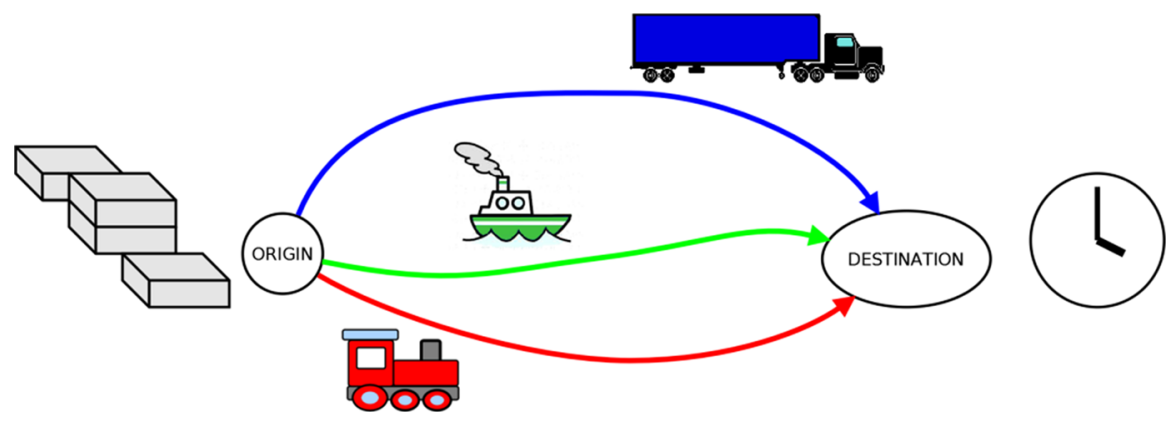

Fig. 1 Basic setting 
component of the assignment, i.e., to use another mode, to use another role, or depart at a different time, as any of those choices will increase his/her own costs.

Despite the simplicity of this system, modelling its performance and functionality in order to study the impact of different policies and decisions is not trivial. In fact, the interplay of different modes and congestion effects make the economic and time-related performance strongly depending on the number of participants/users, and their mode choice. The outcome depends on a variety of factors relating to attitudes (value of time, value of flexibility, etc.), on the costs associated to the trip (duration of the trip, extra costs incurred in waiting for filling up the vehicle) and on the interaction between users, mainly the rules adopted to share the travel costs.

Two main issues are considered only partially in our approach, namely the matching problem (i.e., how to best match trips and demands in time and space, what level of similarity for the routes is acceptable, etc.), and behavioural or organizational challenges related to the perception and desires of the users.

The matching problem (in many variants possible) has been mostly analysed from an operational research perspective. For instance Furuhata et al. (2013) review the matching problem for ridesharing, i.e., matching between a driver and a passenger. Cross-dock operations (Van Belle et al. 2012) are also often modelled as a synchronization or matching problem. Khoi Tran and Haasis (2013) review liner network design that involves matching between (time-dynamic) demand and a timetable of services. Despite a study on how mathematical optimization can be used within an assignment problem is a challenging research direction, it does not fit into the scope of the current paper and is left for future research.

Behavioural and organizational aspects refer to the conditions under which users choose to join an existing travel service, or rely on his/her own. This can be an issue in fostering an uptake of multimodal and intermodal transport links in the freight and logistics worlds (Kreutzberger and Konings 2013). More and more ICT platforms are being set up, facilitating the ease of exchange of information regarding transport demands, and transport supply available. (Port) community systems create possibilities for transport service providers to take into account more up-to-date information regarding current delays and traffic conditions in procedures for planning numbers of transport vehicles required and amounts of crew to be made available. Although technically such platforms seem very promising, effective use of such platforms is only made if sufficiently many transport parties are willing to exchange information. Trust, confidentiality of information, and fairness in costs/ benefits sharing are hereby an issue. These aspects will for the model proposed here be analysed in future research.

We assume that all users in the transportation system have access to a system for publishing demand and offers for capacity, i.e. similar to service centres of infrastructure managers that collect path requests and publish possible opportunities for transportation users. Moreover, everybody has access to truck transportation (own or hired) and can resort to that in any case. This represents the fact that each user, if not able to find a suitable transport supply by train or barge, can always use a truck to have the shipping done. As all demand will always be transported, we are studying an inelastic demand problem, and hence focus mainly on the mode choice analysis. We thus assume that the match can be established on short-notice (a few 
minutes to a few hours from departure). The system that matches up available vehicle capacity on barges and trains and request for vehicle capacity for barges and trains operates on the basis of a First-In-First-Out queue. Regarding the mode choice model, we assume that choices are made such that each traveller chooses the alternative that maximizes his/her utility. Further issues include what each user might find attractive other than pure economic costs, in order to describe a generalized cost function. This includes the value of time, the accepted cost for detour and rescheduling, how the participation to services bundling the demand over larger vehicles and higher economies of scale can be incentivized.

At this stage, we do not consider additional costs not directly related to a single mode choice, such as, for instance, the ownership of a vehicle. This might come from either the fact that ownership is not a problem, i.e., companies might have directly available a fleet of vehicles ready to be used, or that such a fleet can be arranged within a short time, for instance by contacting dedicated shipping companies that can arrange in short term a particular service. This is a growing trend that has been seen in the aggregation of companies into intermodal and multimodal

Table 1 Reference parameters and used values

\begin{tabular}{|c|c|c|c|c|}
\hline Name & Description & Mode & Unit & Default value \\
\hline$L_{\text {truck }}$ & Length of the link per mode & Truck & $\mathrm{km}$ & 120 \\
\hline$L_{\text {barge }}$ & Length of the link per mode & Barge & $\mathrm{km}$ & 120 \\
\hline$L_{\text {train }}$ & Length of the link per mode & Train & $\mathrm{km}$ & 120 \\
\hline$F F T T_{\text {truck }}$ & Free flow travel time per mode & Truck & Hour & 2 \\
\hline$F_{F T T_{\text {barge }}}$ & Free flow travel time per mode & Barge & Hour & 3 \\
\hline$F F T T_{\text {train }}$ & Free flow travel time per mode & Train & Hour & 1.5 \\
\hline$T D$ & Total transport demand & All & Unit & 10,000 \\
\hline$P_{\text {truck }}$ & Price per kilometre per mode & Truck & Eur $/ \mathrm{km}$ & 0.3 \\
\hline$P_{\text {barge }}$ & Price per kilometre per mode & Barge & Eur $/ \mathrm{km}$ & 0.2 \\
\hline$P_{\text {train }}$ & Price per kilometre per mode & Train & Eur $/ \mathrm{km}$ & 0.25 \\
\hline Cap $_{\text {truck }}$ & Vehicle capacity per mode & Truck & Unit/vehicle & 1 \\
\hline Cap $_{\text {barge }}$ & Vehicle capacity per mode & Barge & Unit/vehicle & 160 \\
\hline Captrain $_{\text {tran }}$ & Vehicle capacity per mode & Train & Unit/vehicle & 80 \\
\hline$(a ; b)$ & BPR congestion parameters truck & Truck & None & $(0.15 ; 4)$ \\
\hline$S_{\text {truck }}$ & Saturation flow parameters truck & Truck & Unit/h & 250 \\
\hline$S_{\text {barge }}$ & Saturation flow parameters barge & Barge & Vehicle/h & $(>40)$ \\
\hline$S_{\text {train }}$ & Saturation flow parameters train & Train & Vehicle/h & 6 \\
\hline$\rho_{\text {barge }}$ & Share of cost barge & Barge & None & 1 \\
\hline$\rho_{\text {train }}$ & Share of costs train & Train & None & 1 \\
\hline VoT & Value of time & All & Eur/h & 4 \\
\hline$\alpha$ & Early arrival cost & All & Eur/h & 0.8 \\
\hline$\beta$ & Late arrival cost & All & Eur/h & 2 \\
\hline$\gamma$ & Waiting time cost & All & Eur/h & 0.8 \\
\hline
\end{tabular}


transport service providers (Fazi 2014). Including the costs of ownership would be a further addition to the two time dynamics already considered, namely whether a company should buy or not a vehicle, and introduce further complexity in the mode choice. Finally, some additional objectives are hard to be translated precisely to cost, such as sustainability or flexibility of the transport link. Shinghal and Fowkes (2002) gives an overview and empirical determination of those factors.

\section{Model}

\subsection{Basic notation}

We can now introduce the model. We first define the following parameters and variables that are used in the model. The parameters used are the following Table 1.

A transport demand $T D$ is given, assumed to be divided into a series of generic, interchangeable units, as is common in container transport. This transport demand is related to a single origin and a single destination. It is also assumed that no elasticity effect is present, i.e., all transport demand needs to be moved to the destination. Distance to be covered by each different mode will be a certain $L$, dependent on the mode in general. All transport demand has the same origin and destination. The transport demand is homogeneous also from the point of view of the time, as all demand shares the same time horizon of minimum possible departure time and maximum departure time, and preferred arrival time. Extending this approach to a heterogeneous transport demand is an interesting subject for future research. We consider a single link per mode, i.e., the final network will consider a link per mode per OD, which makes it three links in total. Each link can be seen as the aggregation of multiple successive links that are homogeneous for a particular mode. We discuss the applicability towards networks with more than one link per mode, or multiple origin-destination pairs in Sect. 7.

Every mode over its associated link has a given free-flow travel time FFTT, i.e., the minimum travel time possible, regardless of congestion effects; and a travel related price, $P$, which depends on the distance and the mode. Some factors influencing the travel price such as energy efficiency, technology used, etc., fall outside the scope of this paper and are not considered explicitly here. Moreover, every mode is characterized by a vehicle capacity Cap, which refers to how many transport demand units can be transported in each vehicle. The congestion is depending on a variety of factors that are expressed functionally in the next section, and relate to a main saturation flow per mode, i.e., the maximum amount of vehicles that can be travelling per time unit, plus additional parameters if required (in this case the parameters $a$ and $b$ for the truck link). As for the barge, the actual infrastructure capacity for barge is very high, and its saturation flow is very hard to be reached. In particular, in the experiments proposed later, we find that the maximum flow of barges seen is equal to 40 vehicles/h and the final flow at equilibrium is 23 vehicles per hour. Thus any infrastructure capacity higher than 40 vehicles per hour would yield the same result. 
We assume to be able to describe the costs with the following set of parameters, namely a value of time VoT to translate a delay into a cost, plus a share mechanism that divides the cost for modes in which the vehicle capacity is more than one. This depends on a parameter for barge and train that describes whether the cost allocation is fair among all users ( $\rho_{\text {mode }}=1$, everybody using a vehicle pays a fraction of its cost proportional to the vehicle capacity used), or might favour or disfavour active roles, i.e., the active role (that is always one per vehicle) might pay less or more than each passive role. Respectively, this corresponds to a policy setting in which a stronger time-responsiveness is required, or one where demand will adhere with greater compliance to an external given transport supply. Finally, there are three cost parameters that are used to determine cost of early arrival, late arrivals with regard to a preferred arrival time, and a cost for waiting for the full loading and the departure of a vehicle with a capacity bigger than one.

Additionally, other costs factors can be considered, for instance incentives to particular modes. These cost factors can be represented by a smaller cost per $\mathrm{km}$, or by one-off contributions, or depending on the loading rate of the vehicles. In general, trucks will experience a higher costs as the total operating cost can only be referred to a single discrete transport unit; modes such as train and barge can achieve lower costs if they are fully loaded. Eventual fees, charges, monetary incentive to participate, can be represented in such a framework, additional to the above fixed costs.

\subsection{Variables and functions}

The model is able to deal with multiple time scales, namely the mutual influence of the choice of departure times, congestion and costs for early/late arrival of freight units, and the convergence towards equilibrium. The former dynamic is referred to as within-day (and indexed by the variable $t$ ), as typically transport demand is recurrent and roughly cyclic along period of time such as a day (or a week, or a similar period of time of fixed length, without less of generality). The latter dynamic refers to a process over a long time horizon, every time evaluating the decision takes according the other dynamic. Thus, we refer the latter dynamic as day-to-day, and index it by the variable $d$.

The within-day model works as follows. The users evaluate whether to change mode of travelling, and at what time this decision has to be taken. The departure time for all users should coincide and depend on the trade-off between congestion costs and schedule delay costs. Congestion costs can be dealt with in a different way across modes. Whereas truck traffic congestion may well be dealt with following conventional cost-flow functions such as in vehicular traffic, this decision may not be the most recommended in rail-based and inland navigation systems. In the former, congestion can be considered due to a limited number of paths available per unit of time, primarily due to the use of block sections and scheduling constraints. The latter has normally a much higher capacity than the demand in reality, and thus is a nearly-deterministic mode of transport from this point of view.

The earliest departure times and the travel time for the collaborative modes is moreover a trade-off between the early arrival costs, the queue of requests and 
therefore the waiting time to find a match. So the interesting result of the above model is to find both distribution and rate of mode choices, as well as to find the time window where at equilibrium the highest chance of a prompt match is ensured.

The other time-scale considered in the approach relates to the typical way of finding an equilibrium assignment, which involves modelling the decision of a set of players along a finite amount of time. This basically assumes that the players are repetitively seeking to perform a transport action (say every day), and every time they learn from the past experiences. Each user, after experiencing a certain travel cost at the end of day $d$, decides which combination of (mode, departure time $t$ ) is giving the least expected costs for the next day $d+1$. Via an iterative process of learning the decision (departure time choice and mode) of other users, a series of assignment is found, that ultimately converges to an equilibrium assignment. This point users' costs, expressed as function of mode, travel time and related congestion, departure time and waiting time for bundling sufficient demand in order to fill a vehicle. At equilibrium, for all collaborative modes, active and passive users should match in transport demand offered/requested. Moreover, the costs of all three mode alternatives must be equal.

The main variables of the problem mainly relate to generalized costs experienced, times and flows, and are described in the following Table 2, for each mode.

We model implicitly the amount of vehicles as a decision variable of our model. In fact, active roles act as trigger the start of a service or line, by requesting a new vehicle that can be further used by many other users.

We further consider the within day dynamics as a time-discretized setting.

We next use variables and parameters in a set of functions that determine how attractive a mode and choice of time and role might be. The costs have three major components: a travel-time related component, i.e., the longer the travel time the higher the cost; a distance and mode based component, i.e., for some modes the cost per unit will be lower, and the costs will further shared over a large amount of units; and a delay cost component, that refers to the reliability of the travel time. In other terms, this latter component weighs the possibility to arrive at the preferred arrival time, the costs for being early or late, and some costs associated to waiting in the system. The cost functions per mode, per role, are as follows:

Table 2 Decision variables

\begin{tabular}{lll}
\hline Name & Description & Unit \\
\hline$C_{\text {mode, role }}(t, d)$ & Generalized experienced cost per time $t$ per day $d$ per mode per role & Eur \\
$F_{\text {mode, role }}(t, d)$ & Flow per time $t$ per day $d$ per mode per role & Unit/h \\
$T T_{\text {mode }}(t, d)$ & Travel time after departure per time $t$ per day $d$ per mode & Hour \\
$W T_{\text {mode, role }}(t, d)$ & Waiting time before departure per time $t$ per day $d$ per mode per role & Hour \\
$S D C_{\text {mode,role }}(t, d)$ & Schedule delay cost per time $t$ per day d per mode per role & Eur \\
$N_{\text {mode,role }}(t, d)$ & Cumulative flow per time $t$ per day $d$ per mode per role & Unit/h \\
\hline
\end{tabular}


Truck

$$
C_{\text {truck }}(t, d)=T T_{\text {truck }}(t, d) \operatorname{VoT}+L_{\text {truck }} P_{\text {truck }}+S C D_{\text {truck }}(t, d)
$$

Barge active

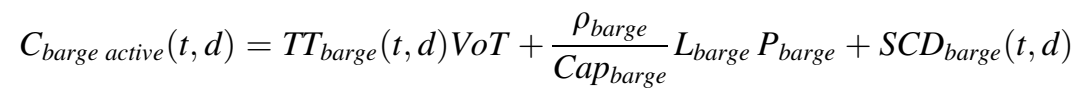

Barge passive

$$
\begin{aligned}
C_{\text {barge passive }}(t, d)= & T T_{\text {barge }}(t, d) \operatorname{VoT}+\left(\frac{1-\rho_{\text {barge }} / \text { Cap }_{\text {barge }}}{\text { Caparge }_{\text {barge }}-1}\right) L_{\text {barge }} P_{\text {barge }} \\
& +\operatorname{SCD}_{\text {barge }}(t, d)
\end{aligned}
$$

Train active

$$
C_{\text {rail active }}(t, d)=T T_{\text {rail }}(t, d) \operatorname{VoT}+\frac{\rho_{\text {rail }}}{\text { Cap }_{\text {rail }}} L_{\text {rail }} P_{\text {rail }}+S C D_{\text {rail }}(t, d)
$$

Train passive

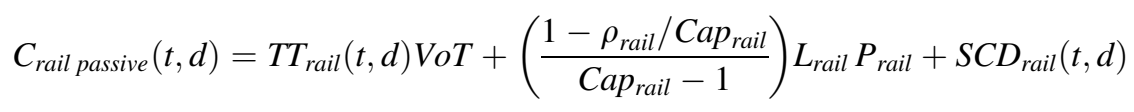

The cost function curves take generally into account that the cost of a trip will increase if the traffic along the link is so high that there will be phenomena of congestion and, due to this, extra travel time is observed. This is due to the travel time function $T T(t, d)$. This is highly mode specific, and explained for the different three modes in the following.

For road traffic, a congestion following a classic BPR-function is used (Bureau of Public Roads 1964), with suitable choice of parameters $\left(a, b, S_{\text {truck }}\right)$ :

$$
T_{\text {truck }}(t, d)=F F T T_{\text {truck }}\left(1+a\left(\frac{F_{\text {truck }}(t, d)}{S_{\text {truck }}}\right)^{b}\right)
$$

In this functional form, $a$ controls the increase in travel time when the number of vehicles in the system reaches congestion at its saturation flow, represented by the parameter $S_{\text {truck }}$. Additionally, the parameter $b$ controls the sensitivity of the system to congestion, i.e., how rapidly travel times increase when the demand is near or above the saturation flow or the infrastructure capacity.

For train, a different travel time function is used, namely a queuing model in which a determined amount of freight paths will be available per unit of time, and the travel time is a fixed travel time plus a waiting time, equivalent to the service time of the queue: 


$$
T T_{\text {rail }}(t, d)=F F T T_{\text {rail }}+\left(N_{\text {rail,active }}(t, d)+N_{\text {rail,passive }}(t, d)\right) / \text { Cap }_{\text {rail }}
$$

Here, $F F T T_{\text {train }}$ represents the travel time in absence of congestion; $N_{\text {train }}$ is the total cumulative flow of train vehicles using the train mode up to time $t$, and Cap $_{\text {train }}$ is the maximum amount of train per hour that can be travelling at the same time on the train link. This assumes a flow of the train network very similar to a queue with a First-In-First-out policy, with fixed travel time, and fixed processing rate. The Travel time does not depend on the role, but rather depends on the amount of vehicles only on the link. Both active and passive roles for the same vehicle will experience the same travel time, apart from the time waiting for loading the full vehicle and eventually depart.

A similar expression holds for barges, with a different vehicle capacity Cap $_{\text {barge }}$ :

$$
\operatorname{TT}_{\text {barge }}(t, d)=F_{F T T_{\text {barge }}}+\left(N_{\text {barge, active }}(t, d)+N_{\text {barge,passive }}(t, d)\right) / \text { Cap }_{\text {barge }}
$$

In practice, the actual infrastructure capacity for barge is very high, and its saturation flow would be almost never reached. In that case, the equation simplifies to a fixed travel time equal to the free flow travel time. Concerning the modedependent costs, the shared mechanism described above is used, with the parameter $\rho$ deciding which share of the costs will be borne by the active users, i.e. those initiating a new vehicle, and the passive ones, i.e. those looking for available spots in existing community services and joining an already organized transport vehicle.

Concerning the Schedule Delay costs, we resort to a commonly accepted treatment in the literature that goes back to the work on bottleneck traffic models of (Vickrey 1969). Basically, a preferred arrival time PAT is given, and early arrivals and late arrivals with regard to the preferred time are penalized by some linear factors, respectively $\alpha, \beta$. On time arrival is in fact of interest in case one wants to increase the reliability of a supply chain link. Then the typical Schedule Delay cost expression can be derived directly for the truck mode:

$$
\begin{aligned}
S C D_{\text {truck }}(t, d)= & \alpha\left(\max \left\{0, P A T-\left(t-T T_{\text {truck }}(t, d)\right)\right\}\right) \\
& -\beta\left(\min \left\{0, P A T-\left(t-T T_{\text {truck }}(t, d)\right\}\right)\right.
\end{aligned}
$$

For collaborative modes, extra waiting time while waiting for filling up the vehicle is a key aspect that is not included in previous studies based on Vickrey's bottleneck model. This is the time spent for bundling enough demand going on the same direction. In general, this time decreases with the amount of units to be transported by the vehicles, as the wider the pool over which demand can be bundled, the easier and faster the process would be. This time is also depending on the ratio of active and passive roles, while the expressions above were independent from the role chosen.

We introduce the concept of waiting time $W T(t, d)$. This is the time span between the time when the unit would be available to be shipped, i.e. loaded, sealed, in place and ready, and the time when the vehicle actually starts is trip. This latter time depends on having the vehicle full. As we assume that only full vehicles can be 
shipped, the time waiting for the actual begin of a trip is actually spent completely to load the vehicle by other transport units.

From the point of view of the schedule delay costs, this waiting time is in fact an extra delay that has to be taken into account while choosing the departure time. Being available for departure earlier increases the chances of an early departure and an on-time arrival. Being ready at the very last minute decreases some waiting costs, as more slack is available to prepare the transport unit, but increases the risk that the unit cannot be boarded on a vehicle and arriving on time. To weight this delay, an additional parameter $\gamma$ is included, that expresses the relative importance for waiting in the system. The extension of the Schedule Delay cost expression for train and barge is thus as follows:

$$
\begin{aligned}
S C D_{\text {rail,mode }}(t, d)= & \alpha\left(\max \left\{0, P A T-\left(t-W T_{\text {rail,mode }}(t, d)-T T_{\text {rail }}(t, d)\right)\right\}\right) \\
& -\beta\left(\min \left\{0, P A T-\left(t-W T_{\text {rail,mode }}(t, d)-T T_{\text {rail }}(t, d)\right\}\right)\right. \\
& +\gamma W T_{\text {rail,mode }}(t, d)
\end{aligned}
$$

In the definition of the waiting time the role (active or passive) is determinant. To this end, we assume that there is commitment, i.e., once a mode is chosen this cannot be changed within the same day; unless no matching is found at the end of the time horizon. This latter case represents the situation when some vehicle is waiting to be loaded, but there will not be enough demand to make it depart full. In fact, transport units will be kept in the queue for a transport vehicle and will not leave the queue unless they are also taking the same decision to switch to a different option. In this case, we consider the possible alternative of by which the vehicles does not depart at the end of the time horizon, and instead the transport units waiting will be transported by trucks at the end of the time horizon. The cost of a truck shipment is incurred. In this case, the choice will determine a different value for waiting time, which will influence the costs, and also push users to adopt a particular role, so that the generalised costs can be minimized. We here re-state that the amount of active roles correspond to the amount of vehicles in the systems. Also, given the assumption of full load vehicles, the ratio between active roles and passive roles (for a given mode) is fixed and determined by the capacity of the vehicles.

\section{Solution process}

The main challenge is to determine an assignment algorithm that allows assigning users to classes and departure times, i.e., allows computing equilibrium in the setting defined above. The underlying mathematical problem is challenging in many aspects, for instance existence, convergence and uniqueness of the equilibrium in assignment is far from trivial, given the fact that user characteristics change when they switch group. The basic problem to be solved is the re-assignment: based on the costs of all other modes and all other roles at one iteration (or day $d$ ), and all relevant time durations, find the best mode, role and time to be chosen for the next day $d+1$. 
We refer to Fig. 2 for a graphical description of the procedure. The simulation of one day $d$, i.e., a within-day dynamics, is reported graphically as a set of tasks to be done iteratively, around the figure. We can start by the item at the bottom of the Fig. 2, going counter-clockwise. Given a subdivision in classes, and departure times, we determine first the time that passive participants in train and barges mode have to spend waiting for a match, $W T_{\text {barge,role }}(t, d)$ and $W T_{\text {rail,role }}(t, d)$. To do so, we consider a cumulative representation $N_{\text {mode, role }}(t, d)$, depicted on the right side in Fig. 2.

The elements $N_{\text {truck }}(t, d), N_{\text {barge,mode }}(t, d), N_{\text {rail,mode }}(t, d)$ represent the amount of participants that have entered the system until time $t$ and at day $d$. All participants choosing for a truck can depart as soon as they enter the system. Instead, $N_{\text {barge,mode }}(t, d), N_{\text {rail,mode }}(t, d)$ include the amount of participants for the two modes that starting offering (for active role) or requesting (for passive role) places on available vehicles at the beginning of time $t$, plus some participants from previous time periods that are still in the process of finding a match (i.e. the queue of requests). As for the matching, we use a FIFO discipline. A user, say the $M$ th train passive user requesting a place on a train will correspond to $N_{\text {rail,passive }}(t, d)=M$, and enter the system at time $t$. We assume that this user has to wait, i.e. the passive users are more than the active users. In this case, this user will have to wait till the

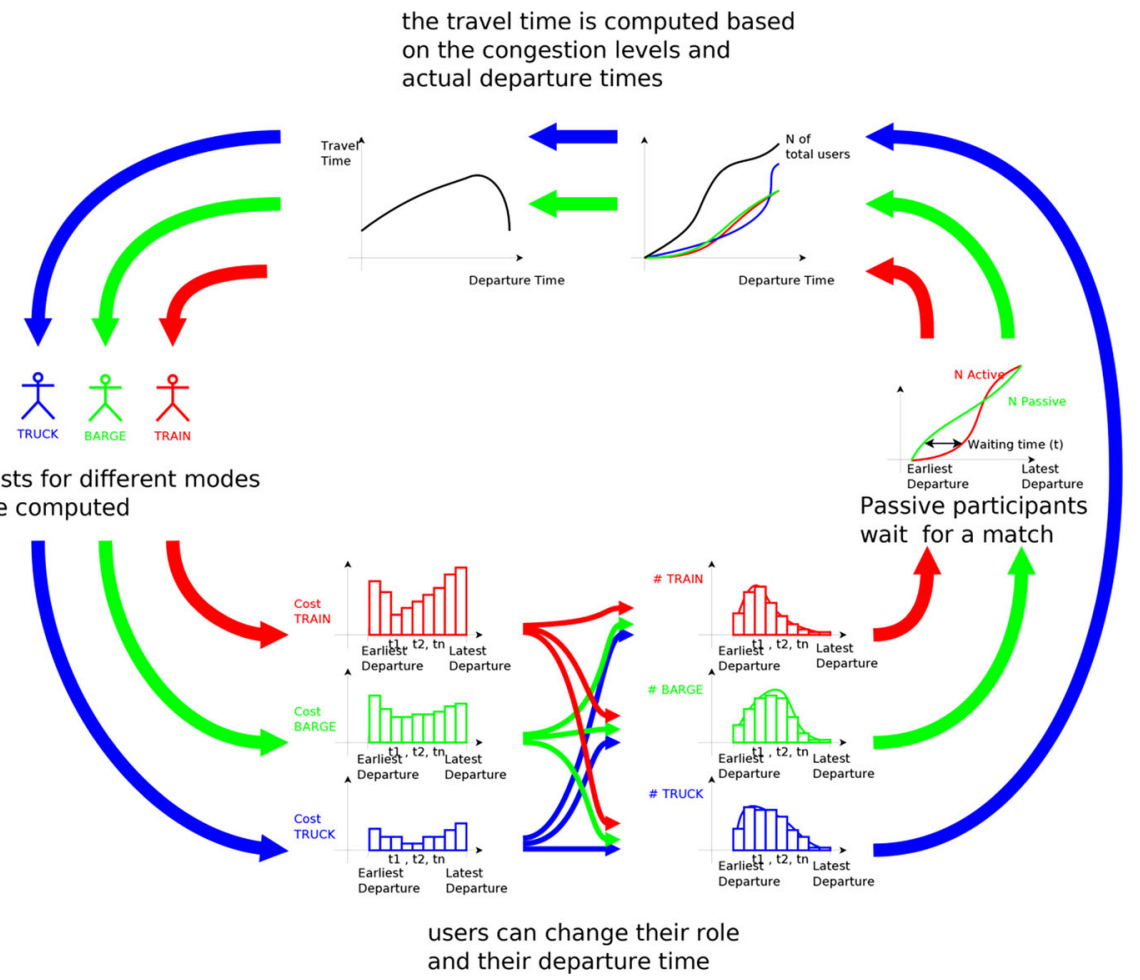

Fig. 2 Graphical illustration of the solution process (Color figure online) 
vehicle identified by $N_{\text {rail,active }}(\tau, d)=M /$ Cap $_{\text {rail }}$ will enter the system at time $\tau$, for a total waiting time of $\tau-t$. The active user identified by $N_{\text {rail,active }}(\tau, d)=$ $M /$ Cap $_{\text {rail }}$ will have a waiting time of 0 . In the opposite case, when passive users are more than active ones, i.e. transport units are waiting for vehicles, the reasoning is analogous and opposite. The waiting time will then be 0 for the passive users, and a similar expression for the active users.

Some extra detail of the matching process for collaborative mode is reported in Fig. 3. The Figure refers to barges, but for railways, the process is exactly analogous. In the picture, the $\mathrm{x}$-axis represents times, starting from an earliest possible departure time (i.e. nobody perceives departing earlier attractive, for a given Preferred Arrival Time) and going to a latest possible departure time (departing after which will result in excessive scheduling delays even in free flow conditions). The $\mathrm{y}$-axis are the cumulative flows of barge users. Two curves are plotted Cap barge $\times N_{\text {barge,active }}(t, d), N_{\text {barge,passive }}(t, d)$ for a given day $d$, respectively in purple and yellow. Those curves represent respectively the cumulative amount of passive transport units amount that have entered the system, and are available for matching; and the amount of vehicle capacity for extra units to be carried. The minimum of the two curves, in other terms, the lower envelop of the curves, represents the transport units on vehicles that have been fully loaded and can actually depart. This latter correspond to the vehicle flow rate at time $t$ during day $d$, that is used among other things to determine the congestion and the travel time. The step-like behaviour of Cap barge $_{\text {barge, active }}(t, d)$ originates from the discrete and large vehicle capacity of a barge; every step corresponds to a new barge available, i.e. Cap $_{\text {barge }}-1$ new places available for shipment. The one unit to be subtracted is for the active role, which triggers the usage of the barge.

The two shaded areas correspond to the cases in which there are more active barge users than passive users (i.e. barges are waiting to be filled up) in green, at the left of the Fig. 2; and the case in which there are more passive users than active ones, i.e. there is a queue of transport units to be loaded over a limited amount of

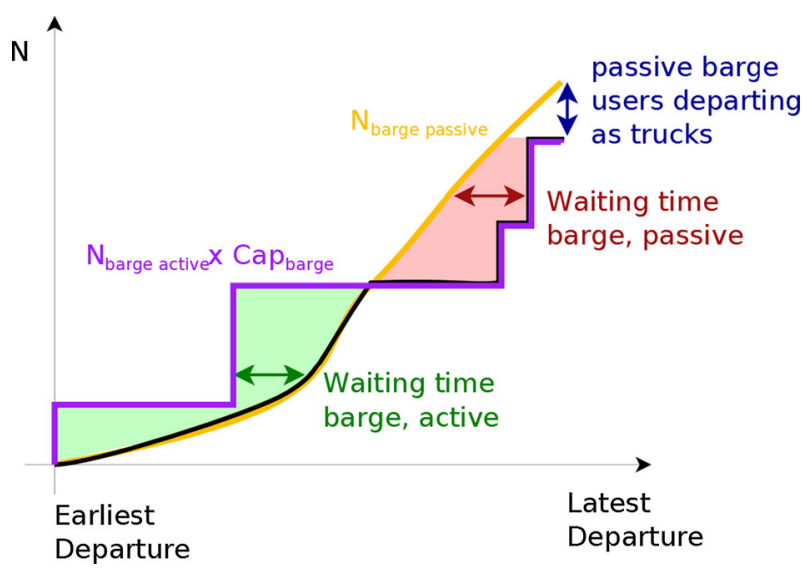

Fig. 3 Example of a matching process for barge users (Color figure online) 
barges, in red, top-right. To those two situations we can associate waiting time, also reported in Fig. 2. The final gap between the number of active barge users and passive barge users at the latest acceptable departure i.e. the difference highlighted by the vertical blue arrow in the Fig. 2, corresponds to participants (in this case passive) that will not be able to fill a vehicle and need to be transported in other ways. They might depart as trucks, or share the extra costs of the partially full vehicle. The matching mechanisms works analogous for the railway mode, with a different parameter for $\mathrm{Cap}_{\text {rail }}$. Once the amount of vehicles is determined, the travel time per mode, and the total travel time can be computed, based on the link congestion dynamics reported in the previous section.

Each user, after experiencing a certain travel cost at the end of day $d$, decides which combination of (mode, departure time $t$ ) is giving the least expected costs for the next day $d+1$. This a dynamics that is named day-to-day, and basically represents a learning process on the decision of other users. This corresponds to the left part of Fig. 2. The basic problem to be addressed is changing mode, changing role, and changing departure time.

The problem is solved by considering that the new fraction of flows at each time period chooses the least costly alternative, by means of a logit model. Logit models describe the probability of choosing a certain mode alternative as function of the expected cost differences. They explicitly consider stochasticity in this decision caused by uncertainty in future costs, error perceptions and heterogeneity in the value of times. The stochastic nature of the costs, typical in traffic assignment, is also easily justifiable in freight transport. Different descriptions can be considered for the logit function used for mode choice and the departure time choice, taking into account the different elasticity of the users to switch mode or transport or adjust departure time from 1 day to the other. In this study we use the simple Multinomial Logit Model (Ben Akiva and Lerman 1985):

$$
P\left(\text { mode } \_i\right)=\frac{e^{-C_{\text {mode }}-^{i}(t, d)}}{\sum_{j} \sum_{\tau} e^{\left.-C_{\text {mode }}\right\lrcorner^{j(\tau, d)}},}
$$

where the probability of choosing a certain mode alternative $i$ at time $t$ on a day $d$ is dependent on the cost of $i$ in comparison to the cost of all other alternatives and at any time period $\tau$.

To simulate smoothing effects from incremental information acquisition and learning we adopt the traditional Method of Successive Averages, which is common in the literature for calculating the assignment process (Jourquin and Limbourg 2006). The MSA adopted calculates at every iteration the new costs given the mode choices from the previous day, and updates the mode choices for the current day, but swapping a part of the flows in inverse proportion to the number of days run in the simulation. We call this a flow averaging (FA) criterion.

There is a variety of other approaches for assigning goods to the different mode alternatives, and for modelling the convergence towards equilibrium. Different methods have been considered and evaluated (e.g., deterministic assignment, dynamic swapping algorithms), which however did not add insight or enriched the analysis of this paper, and therefore their results are not included in this paper. To 
take into account that the elasticity of adapting the scheduling times may be different than switching mode, we consider different flow averaging parameters for the two choice levels. The procedure goes along the following operations:

Given the cost vector for day $d$

Calculate mode choice probabilities

Calculate total flow amount per mode

Calculate earliest departure time choice probabilities

Perform an MSA-FA and update flow observed at $d$ to flow at day $d+1$

This procedure is iteratively performed by again computing the flows on links, associating a cost, and so on, along the Fig. 2. At the end of iterative procedure a solution is found, in which the all the decisions of the users (departure time, mode, role) are in equilibrium. i.e. no user has incentive to change its choice to get a minor cost. This final solution is the result of a few factors. Every day, the users evaluate whether to change mode of travelling, and at what time this decision has to be taken. The departure time for all truck users should coincide and depend on the trade-off between congestion costs and schedule delay costs. The earliest departure times and the travel time for barge and rail is moreover a trade-off between the early arrival costs, the queue of requests, the waiting time to find a match and the travel time on the link due to the restricted infrastructure capacity.

So the output of this model is the simultaneous determination of mode choice, as well as to find the time window where at equilibrium the highest chance of a prompt match is ensured. Having more users choose for the rail and barge increases the chance of match, but also increases the amount of time lost due to congestion along the link. At equilibrium, the costs of all three mode alternatives must be equal, or an alternative should be considered by the users These effects can be graphically seen in the next section where they are analysed when discussing Fig. 4.
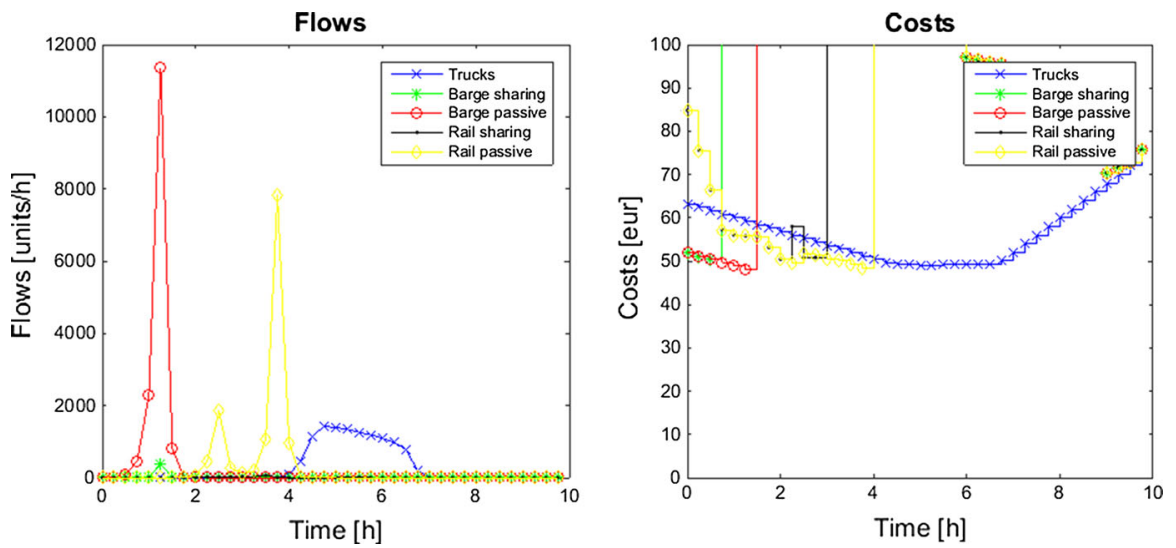

Fig. 4 Choice of departure time in the resulting model: flows (left), costs (right) 


\section{Experimental analysis}

We apply the innovative model presented on a simple test case to show the applicability and the outcomes. A systematic analysis can provide insights into the factors determining increase/decrease in modal share, and which policies can be used to promote further rail and barge in a multimodal transportation market. The overall model is used to study the interplay between costs (dependent on the role chosen), departure time (i.e. possible time spent waiting for a match, plus experienced travel time) and congestion levels and waiting time experienced along the link.

Again, we used the basic case reported in Fig. 1, with a single link per mode, and a single origin-destination pair. We also used the values reported in Table 1 when not differently reported. The model and solution algorithm here sketched have been translated to software code, and implemented in Matlab R2014. The computation for the simple single-origin destination, one link per mode, $10 \mathrm{~h}$ time horizon, 200 iterations of assignment, is performed within two seconds on a standard desktop computer.

We analyse in Fig. 4 the equilibrium solution, in terms of flow per departure time (left plot), generalized costs (right plot). For every plot, the departure time of the transport unit time is on the $\mathrm{x}$-axis, i.e., the time at which the transport unit will start its trip towards the destination; flow (respectively cost) on the y-axis. Concerning the flows, one can see how the vast majority of the flow can be transported via collaborative modes such as barges and rail. The final modal share is $39 \%$ (barge), $33 \%$ (rail), $28 \%$ (truck). Note that this share is largely influenced by the

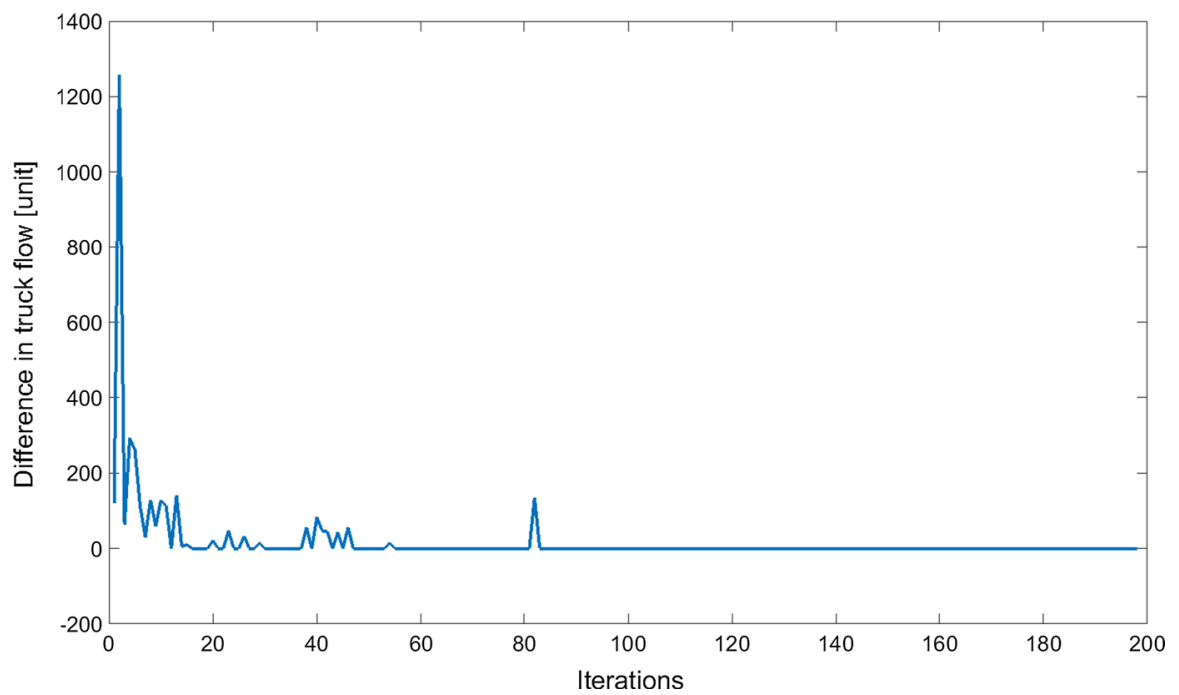

Fig. 5 Difference between maximum flow by truck, across iterations (x-axis), when no information is present versus when information about queue length is given 
parameters chosen. We present in this section and the following a sensitivity analysis of the results, and a discussion on how to find realistic parameter values.

The different speed and time dynamics of the different modes result in different peaks in departure times: this is between time 1 and 2 for the barges; trains feature a smaller travel time and a lower vehicle capacity, and result in a peak at later times, between 2 and 4 . Also note that due the restricted infrastructure capacity available on the rail, there are two peaks. The final peak of the trucks is even later, with the maximum around 5. Looking only at the time of the peaks, and not at the total flow per mode, it is mainly the responsiveness of the mode to determine the time of the peak, and not the average travel time, as the speed for train is higher than for trucks. The costs (Fig. 4, right) report that the different modes have competitive costs at different times; and that the peaks in flows corresponds to those intervals where a mode is cheaper in terms of generalized costs than another one, respectively barge, rail, truck. This is a typical outcome at equilibrium.

The subject of a second evaluation is the sensitivity to the information policy. We consider a case in which users will be able to know that a mode will result in no match at the end of the time horizon, and have thus the possibility to choose the truck earlier. It turns out that despite the modal share and the flows are different through the iterations, the learning mechanisms (day-to-day) is able to incorporate this, and the resulting equilibrium is actually the same. We report in Fig. 5 the iterative procedure of convergence, namely the difference between the maximum flow by truck (i.e., a measure of congestion), on the y-axis, from day 0 (i.e., the first day) to day 200 (when the procedure converges), on the x-axis. The amount of

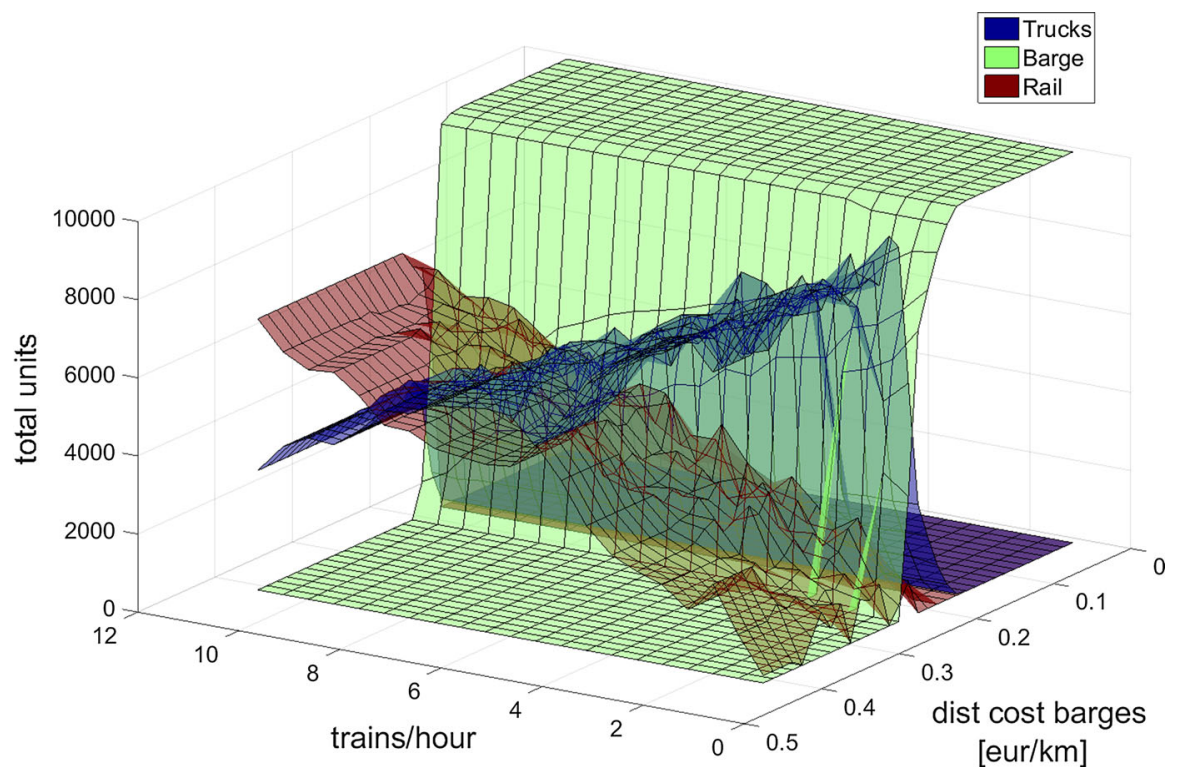

Fig. 6 Total units and modal share as influenced by rail infrastructure capacity, and the distance-related costs of barge 
fluctuations relate to the algorithmic procedure of convergence. The difference is always positive, i.e., when information is provided the amount of trucks on the road are always less.

We finally study the sensitivity of the final assignment equilibrium, on multiple parameters. For sake of illustration we limit our presentation the interplay of trains/ h, i.e. infrastructure capacity of the railway link, in terms of trains per hour allowed, compared with the distance cost of the barges, i.e., cost per $\mathrm{km}$. Such a sensitivity study is interesting for instance to determine to which extent the modes of rail and barge are substitute of each other, and favouring one (by means of fiscal incentives, or improved link flow) will attract mostly users from the other collaborative mode, or instead will result in a decrease of the truck movements.

To this end, Fig. 6 reports the share of flows in the three different modes as resulting from the interplay of those two parameters. From the figure, it is evident how the increase in the railway infrastructure capacity (for instance by building a dedicated railway line, and with an improved signalling system of the railway, as done in the Betuwe Route project in the Netherlands) allows a shorter travel time by rail, and thus higher volumes in the rail link (left-top area). On the other hand, if barge costs are made very competitive by some kind of fiscal incentive (left area) then the vast majority of the flows will be transported by barge. In this case the time dynamics and the capacity of the barges and the waterway infrastructure is such that barges can actually achieve a very high modal share, depending on the incentive. On the other hand, even the highest railway infrastructure capacity considered, i.e., 10 train paths per hour, or a freight train every $6 \mathrm{~min}$, cannot attract all demand to the railway mode, and trucks are still used. By providing exact parameter and calibrate them to the actual freight flows, a possible application of such a system might be the evaluation of infrastructure and economic decisions in the context of a multimodal freight network.

\section{Applicability to general networks}

The model described so far has been presented using rather simplifying assumptions, with its relevance demonstrated on a small study case. Being a relatively innovative approach, our interest lied in showing the main modelling characteristics rather than its actual applicability in realistic case studies. We discuss in this section the possibility to extend the theoretical model to general networks with realistic and complex topology, and realistic flows. Further research should address the translation of these recommendations into actual implementation in models and software packages usable for stakeholders.

\subsection{Realistic networks}

The network with a single link per mode should be extended first to consider multiple links connecting each origin and destination, and multiple origin and destinations; trans-shipment along the network is not considered here; congestion 
propagation effects across links are also not modelled. We deal first with the case of networks in which the assignment is performed for a single mode and multiple links.

In this case, established techniques from traffic assignment can be used; which involve finding an implicit (node based or bush-based) or explicit (route-based) set of origin-destination paths. More details on those models can be for instance found in Tampère et al. (2010), Dial (2006) and Gentile (2014). A logit model (or other random utility models) determines the flow per path and per time interval based on the travel time of a path and the available alternatives. Moreover, the travel time per link per time interval is considered based on the actual (time-dependent) flow. Considering multiple origins and destinations will determine additional congestion, and its characteristic back-propagation on the links and across the nodes, but this issue has already been dealt with to an established state of the art in Dynamic Traffic Assignment. Computational speed and mathematical quality of those approaches do also not present particular challenges (Dial 2006; Gentile 2014).

\subsection{Complex networks}

Modelling the trans-shipment of goods along the network (and not a complete modal choice for the origin-destination pair) is a further step forward. This is similar to multimodal transport assignment in public transport where cars or bikes can be used to reach a railway station and vice versa. Fundamentally different from those approaches is the fact that a mode choice for a leg does not imply a mode choice for another leg, i.e., if I go by car to the station I need to go back by car from that station. In freight traffic, one can assume that bundling and consolidation at the level of logistic operators make available vehicles at all nodes.

We assume that in this case some dynamics are inherently different due to the different modes have to be coupled. By simplification and using so-called bushbased or route-based algorithms, the problem can be translated to a simpler network in which there is a particular (feeder) mode used, a transhipment activity; a (connected) mode used. This is also common in DTA literature, where a general approach is to approximate multi-commodity flow network models by using singlecommodity approaches. In that case, the flow and congestion in the first and last link are mode-dependent and can be tackled in the general manner proposed in the example of this paper. The transhipment activity can be modelled as an additional time duration, which is made up of a fixed time (related to the unloading/movement, loading of goods) plus a synchronization time, which depend on the departure time of the connected mode. This latter process can be determined in an analogous manner as the departure time choice of the barge and rail mode in the example proposed in this paper.

\subsection{Calibration}

The realistic outputs of the model depend on a variety of parameters, which affect the final results (modal share, flows, costs) to a certain extent. Calibration of relatively large set of parameters can be performed based on the observed flows for a given link (saturation flow, travel time), possibly integrating planning or 
operational data (for barges, railways, possibly trans-shipment). Practical approaches such as those sketched in Jourquin (2005) or Zhang et al. (2013) are here of interest. Determining precisely the VoT of goods and the responsiveness to time, in the form of early cost, delay costs and additional penalties for transshipment or mode choice can be determined by stated preference data, revealed preference data, or policy guidelines. Policy incentives and implications, organizational factors, commitment of players towards mode choice, integration in the value chain of the all logistic service (more or less storage needed, additional variability of deliveries,...) should also be taken into account when calibrating the flows observed with those simulated.

\section{Conclusions and future research directions}

This paper proposes a Dynamic Traffic Assignment model for multimodal freight networks. In freight networks, it is economically attractive to bundle demand over modes that have large vehicle and infrastructure capacity such as barges or rail. Moreover, this helps reaching the modal share (relating congestion and environmental constraints) set by policy rules. The key problem solved is the determination of expected freight volumes over different modes for planning and policy studies, given some demand and parameters determining the dynamics of the different modes.

The model proposed makes it possible to consider those modes in a more detailed way compared to existing assignment models for freight networks. A key innovative feature is the intrinsic consideration of time-varying aspects. This relates to the time responsiveness in the assignment, the impact of congestion over links when peak demand is travelling, and the synchronization of transport units using modes that have large vehicle capacity (such as barge and rail). We consider two different time dynamics: there is a learning dynamic over a long amount of rounds (days); and a departure time and mode choice within a given time horizon (within-day). In the model the generalized costs of modes varies over time, i.e. different modes can have different attractiveness at different times compared to a preferred arrival time at destination, due to congestion, synchronization constraints, bundling time. This is has paramount importance in demand-responsive logistic services, which strongly vary their costs in time, and are characterized by different time-dependent parameters and constraints. This results in what would be called a within-day dynamic intertwined with a day-to-day equilibrium in Dynamic Traffic Assignment terms (Tampère et al. 2010). In fact, an ambition of this work is to start bridging the gap between the relatively sophisticated models used for car traffic assignment and the simplified ones that are still characteristic of freight.

We evaluate the model over a small theoretical network with multiple modes, analysing sensitivity of the result to various parameters and incentives that policy makers might consider, and studying the impact of different information sharing polices, and the possible impact of cost incentives towards modal shift to barge or rail from trucks. The approach is able to study the interrelation of multiple factors and can determine a large region where a particular mode can be interestingly 
competitive with the other ones. From a policy perspective, the consequences of a large set of incentives and constraints can be evaluated, and it is possible for instance to determine to which extent rail and barge are competing against each other, instead of taking over the modal share of the trucks.

Even though some of the assumptions used might need to be further study in order to enhance the direct applicability of the model, we believe that this is a preliminary investigation; and more realistic characteristics could be incrementally included in future works. The integration of many operational aspects in planning decisions, which would focus on the availability and quality of multimodal links, impact of information sharing policies, as well as the possibility to define synchronization of multiple modes along intermodal links might make the evaluation of those latter factors more precise and reliable. In fact, the next steps in this research will be to extend the study to (1) include unreliability in travel time, test the impact over more complex networks where transhipment can occur, and thus intermodal and synchromodal freight could be analysed; (3) evaluate the impact of commitment and pre-reservation in the market for matching available demand and vehicle capacity, as an additional decisional layer; (4) consider a gametheory setting where the price paid is part of a bidding mechanism and can be further differentiated between different category of users and finally (5) consider realistic test cases to show the applicability to larger cases.

Acknowledgments This research is supported by the Port Research Center project "Innovative Concepts for Inter Terminal Transport on Maasvlakte 1 and 2 at the Port of Rotterdam" and partially supported by the VENI project "Intelligent multi-agent control for flexible coordination of transport hubs" (Project 11210) of the Dutch Technology Foundation STW.

Open Access This article is distributed under the terms of the Creative Commons Attribution 4.0 International License (http://creativecommons.org/licenses/by/4.0/), which permits unrestricted use, distribution, and reproduction in any medium, provided you give appropriate credit to the original author(s) and the source, provide a link to the Creative Commons license, and indicate if changes were made.

\section{References}

Agatz NAH, Erera A, Savelsbergh M, Wang X (2012) Optimization for dynamic ride-sharing: a review. Eur J Oper Res 223(2):295-303

Bell MGH, Liu X, Rioult J, Angeloudis P (2013) A cost-based maritime container assignment model. Transp Res B 58:58-70

Bell MGH, Liu X, Angeloudis P, Fonzone A, Hosseinloo SH (2011) A frequency-based maritime container assignment model. Transp Res B-Meth 45(8):1152-1161

Ben Akiva M, Lerman S (1985) Discrete choice analysis. MIT Press, Cambridge, MA

Bock S (2010) Real-time control of freight forwarder transportation networks by integrating multimodal transport chains. Eur J Oper Res 200:733-746

Bureau of Public Roads (1964) Traffic assignment manual. U.S. Dept. of Commerce, Bureau of Public Roads, Office of Planning, Urban Planning Division, Washington

Cantarella GE (1997) A general fixed-point approach to multimode multi-user equilibrium assignment with elastic demand. Transp Sci 31(2):107-128

Dial RB (2006) A path-based user-equilibrium traffic assignment algorithm that obviates path storage and enumeration. Transp Res B 40:917-936 
European Commission (2001) White Paper: European transport policy for 2010: time to decide, Brussels Fazi S (2014) Mode selection routing and scheduling for inland container transport, Ph.D. Thesis, Technical University Eindhoven and Dinalog

Friesz TL, Gottfried JA, Morlok EK (1986) A sequential shipper-carrier network model for predicting freight flows. Transp Sci 20(2):80-91

Furuhata M, Dessouky M, Ordóñez F, Brunet M-E, Wang X, Koenig S (2013) Ridesharing: the state-ofthe-art and future directions. Transp Res Part B 57:28-46

Gentile G (2014) Local User Cost Equilibrium: a bush-based algorithm for traffic assignment. Transp A Transp Sci 10:15-54

Ghoseiri K, Haghani A, Hamedi M (2011) Real-time rideshare matching problem. University of Maryland, Department of Civil and Environmental Engineering, UMD-2009-05

Guelat J, Florian M, Crainic TG (1990) A multimode multiproduct network assignment model for strategic planning of freight flows. Transp Sci 24(1):25-39

Harker PT (1987) Predicting intercity freight flows. VNU Science Press, Utrecht

Huang H-J, Yang H, Bell M (2000) The models and economics of carpools. Ann Reg Sci 34:55-68

Jourquin B (2005) A multi-flow multi-modal assignment procedure on large freight transportation networks. Stud Reg Sci 35:929-945

Jourquin B, Beuthe M (1996) Transportation policy analysis with a geographic information system: the virtual network of freight transportation in Europe. Transp Res C 4(6):359-371

Jourquin B, Limbourg S (2006) Equilibrium traffic assignment on large Virtual Networks: implementation issues and limits for multi-modal freight transport. EJTIR 6(3):205-228

Khoi Tran N, Haasis H-D (2013) Literature survey of network optimization in container liner shipping. Flex Serv Manuf. doi:10.1007/s10696-013-9179-2

Kreutzberger E, Konings R (2013) Twin hub network: an innovative concept to boost competitiveness of intermodal rail transport to the hinterland. In: Proceedings of the Annual meeting of the Transportation Research Board, Washington, DC

Maia LC, do Couto AF (2013) An innovative freight traffic assignment model for multimodal networks. Comput Ind 64:121-127

Newton S (2008) WORLDNET: applying transport modeling techniques to long-distance freight flows. In: Association for European Transport Conference 2008, Strasbourg

Peeta S, Ziliaskopoulos AK (2001) Foundations of dynamic traffic assignment: the past, the present and the future. Netw Spat Econ 1:233-265

Qian Z, Zhang M (2011) Modeling multi-modal morning commute in a one-to-one corridor network. Transp Res Part C Emerg Technol 19:254-269

Shinghal N, Fowkes AS (2002) Freight mode choice and adaptive stated preferences. Transp Res Part E 38:367-378

SteadieSeifi M, Dellaert N, Nuijten W, Woensel T, Raoufi R (2014) Multimodal freight transportation planning: a literature review. Eur J Oper Res 233:1-15

Tampère CMJ, Viti F, Immers L (2010) New developments in transport planning: advances in dynamic traffic assignment. Edward Elgar Publishing, Cheltenham

Tavasszy L (1996) Modelling European Freight transport flows. Ph.D. Thesis, Delft University of Technology, the Netherlands

Van Belle J, Valckenaers P, Cattrysse D (2012) Cross-docking: state of the art. Omega 40(6):827-846

Vickrey WS (1969) Congestion theory and transport investment. Am Econ Rev 59:251-260

Viti F, Corman F (2013) Equilibrium and sensitivity analysis of dynamic ridesharing. In: Proceedings of the 16th IEEE-ITS conference, The Hague, The Netherlands

Viti F, Corman F (2014) A joint day-to-day mode and within-day departure time choice model for the analysis of dynamic ridesharing. In: Proceedings of the 5th DTA symposium, Salerno, Italy

Viti F, Corman F, Tampère C (2012) Ridesharing: equilibrium model and case studies. In: Proceedings of the 17th International Conference of Hong Kong Society for Transportation Studies, 15-17 Dec 2012, Hong Kong

Viti F, Tampère CMJ (2010) Dynamic Traffic Assignment: recent advances and new theories towards real time applications and realistic travel behaviour. In: Tampère CMJ, Viti F, Immers LHB (eds) New developments in transport planning. Edward Elgar, Cheltenham

Wardrop JG (1952) Some theoretical aspect of road traffic research. ICE Proc Eng Div 1(5):767. doi:10. 1680/ipeds.1952.11362 
Zhang M, Wiegmans B, Tavasszy L (2013) Optimization of multimodal networks including environmental costs: a model and findings for transport policy. Comput Ind 64(2):136-145

Francesco Corman is Assistant Professor at the Section of Transport Engineering \& Logistics, Delft University of Technology. His research career started in Italy at Roma Tre University, he got a Ph.D. at Delft University of Technology and Post-Doc at Katholieke Universiteit Leuven, Belgium. He has authored or coauthored more than 100 peer reviewed contributions to international journal or conferences, and more than 15 papers in high impact journals. His research interests include optimization in discrete transport systems, railway traffic control, scheduling of discrete event systems, study of dynamics of uncertainty in transport systems.

Francesco Viti is currently Associate Professor at the University of Luxembourg. He obtained a Ph.D. at the Delft University of Technology and worked as Research Associate at KU Leuven, where he is still visiting professor. His research topics include Transport Modelling and Planning, Mobility Demand Analysis and Management, Mobile Sensor Networks, Network Demand Estimation, Intelligent Transportation Systems. He published more than 40 journal articles and 120 papers and book chapters. He is currently Associate Editor of the Journal of ITS and of Transportation Research Part C.

Rudy R. Negenborn received the Ph.D. in distributed control from Delft University of Technology, The Netherlands. Currently, he is an associate professor in control for transport and logistics at the Transport Engineering and Logistics Section of the Department of Maritime and Transport Technology, Delft University of Technology. His research interests include multiagent systems, distributed control, and model predictive control, with applications in flow control in transportation networks, intermodal transport networks, and transport over water. 